\title{
Impact of fetal and neonatal malnutrition on the onset of puberty and associated noncommunicable disease risks
}

This article was published in the following Dove Press journal:

Adolescent Health, Medicine and Therapeutics

2 February 20II

Number of times this article has been viewed

\author{
Nicholas E Connor \\ Child Health Research Foundation \\ Dhaka Shishu Hospital, Dhaka, \\ Bangladesh
}

Correspondence: NE Connor, MSc ANISA Project Coordinator Child Health Research Foundation Dhaka Shishu (Children's) Hospital, Sher-E-Bangla Nagar, Dhaka - I207, Bangladesh Fax 880-2-9I 28308

Tel + 8801758248480

Email connorne@icddrb.org
Background: Fetal and neonatal malnutrition impacts the timing of the onset of puberty. The timing of puberty onset has been shown to be a rough indicator of noncommunicable disease (NCD) risk. Recent advances in understanding the various inter-related neurochemical and genetic controls underpinning puberty onset have shed new light on these interesting and important phenomena. These studies have suggested that developmental trajectory is set very early by epigenetic mechanisms that serve to adjust phenotype to environment.

Objective: The aims of this article are to review the most recent research into the proximate mechanisms that initiate puberty; to explore how the activation of those mechanisms could be affected by nutritional cues received during fetal and neonatal life; and, finally, to briefly explore the ramifications for public health.

Methods: An extensive literature review was performed using PubMed (1950 to September 2010) and Google Scholar (1980 to September 2010) using the search terms "puberty onset", "perinatal", and "neonatal malnutrition". English language, original research, and review articles were examined; pertinent citations from these articles were also assessed.

Results: Literature detailing biochemical pathways and evolutionary explanations of human puberty itself led quickly to a noteworthy connection between neonatal malnutrition, puberty onset, and NCD risk. A strong connection was found between maternal malnutrition during critical windows (followed by catch-up growth in childhood) and an accelerated onset of puberty. Children subject to early nutritional insult not only are likely to undergo puberty earlier but also show an increase in their risk of developing NCDs in later life. Several authors have suggested that this relationship may show potential as an early proxy indicator of susceptibility to these types of diseases, which are an increasing concern in both affluent and developing countries.

Conclusions: More attention should be paid to fetal and neonatal nutrition and puberty onset if we are to meaningfully curb the troubling growth of NCDs now and in the future. Efforts to improve maternal conditions among vulnerable poor groups as well as more affluent groups worldwide should be explored, especially among groups undergoing rapid nutritional transition.

Keywords: malnutrition, early puberty onset, IUGR, maternal health, maternal capital, NCD risk, kisspeptin, leptin, fetal programming

\section{Introduction}

The processes underpinning both early growth and timing of adulthood have undergone significant selective pressures for the entirety of our evolutionary history. ${ }^{1}$ Recent findings indicate that the timing of puberty onset is affected by nutritional insults occurring during certain early critical development windows. Maternal and early life nutritional cues seem to 'program' the developmental trajectory of a child 
by augmenting the timing of puberty onset for reproductive advantage in a predicted poor environment. These augmentations to reproductive strategies also increase many adult metabolic health risks. ${ }^{2,3}$ Understanding the molecular mechanisms underpinning these related phenomenon is important, as precocious puberty is itself linked with various negative and costly outcomes, including heightened risk of developing complex and costly noncommunicable diseases (NCDs) in adulthood.

From a public health standpoint, serious health risks arise when metabolic programming is unmatched with adult nutritional environment. When we consider poor maternal effects, serious risks may even echo beyond the first generation. ${ }^{4,5}$ Early onset of puberty and adult NCD risk may very well share common developmental origins. In our time of overconsumption, expanded migration, and rapid urbanization, understanding the hidden links between neonatal malnutrition, puberty onset, and chronic disease susceptibility may prove to have a two-fold benefit: first, to allow better identification of individuals with heightened NCD risk and, second, to better inform thinking and policies regarding investment in the health resources of mothers and young children as the most effective disease prevention investment over the short, long, and very long term. ${ }^{6,7}$

Using an evolutionary framework, this article reviews the latest findings regarding nutrition in early life and the timing of puberty onset. It begins with proximate causes of the onset of puberty and investigates the more discrete genetic and epigenetic mechanisms, in order to best address how early life events calibrate pubertal onset after a long period of dormancy. This is followed by a brief discussion of different forms of malnutrition, moving to fetal and neonatal malnutrition. Then, how critical fetal programming periods can permanently alter metabolic and reproductive strategy through epigenetic programming is examined. In considering the impact of early nutritional cues throughout the life course, it is important to understand the potential maladaptive effect these early set mechanisms have in our rapidly changing modern societies. Finally, the vital importance of adequate maternal and early childhood nutrition as a fundamental concern in a comprehensive approach to long-term global health efforts will be discussed.

\section{Puberty onset}

The onset of puberty is the watershed event in an individual's developmental trajectory. It is important to recognize that in comparison with other mammals, including our close primate relatives, Homo sapiens undergo an extended juvenile period preceding the onset of puberty. This characteristically long delay exposes the individual to an extremely high risk of prereproductive mortality. This conspicuous risk must therefore be offset in some manner if the juvenile stage is to be evolutionarily advantageous. It has been theorized that this long delay is accounted for by increased communal investment by close kin and other allies. This pattern, which is found among other social animals, allows for optimal development of our complex brains and provides the considerable time needed for learning of information and skills crucial to survival, development, and transmission of language and social skills, as well as achieving adequate body size required for longevity. ${ }^{1,8}$ Developing into a close-knit member of a society confers many reproductive advantages to a population over and above the usual rapid mammalian growth and reproductive trajectory. It follows that if poor neonatal nutrition accelerates this process (changing reproductive strategy), it must confer comparable adaptive benefits in terms of reproductive success. Unfortunately, accelerated puberty may not be congruent with sociological expectations or psychosocial maturation in an increasingly complex society. ${ }^{9}$

Mammalian puberty is neurochemically initiated by the marked increase in the pulsatile release of gonadotropinreleasing hormone $(\mathrm{GnRH})$ from the preoptic anterior hypothalamus. ${ }^{10}$ The continual GnRH pulse then drives development and maintains the eventual reproductive functioning of the secondary sex characteristics via the subsequent release of follicle-stimulating hormone and luteinizing hormone, which in turn control the levels of the gonadal steroids: testosterone in males and estradiol in females. This chemical cascade physically manifests in the first appearance of breast buds in girls and genital changes in boys. According to Marshall and Tanner, the mean (standard deviation) initiation of puberty occurs in girls at $11.15( \pm 1.10)$ years and in boys at $11.64( \pm 1.07)$ years. ${ }^{11,12}$ Several other studies on more representative populations have arrived at concordant figures. ${ }^{13}$ Co-ordination of these processes results in the gradual development and maintenance of sexual characteristics throughout life, allowing for reproductive competence.

The timing of GnRH pulsatile release is dependent on the co-ordinated increase of a great number of inter-related and complex excitatory stimuli and functioning of various receptors, including but not limited to leptin, kisspeptin, neurokinin B, and glutamate alongside various glial small cell-cell signaling molecules. These increasing processes are paired with loss of other trans-synaptic inhibitory signals within the arcuate nucleus located in the mediobasal hypothalamus; these processes all feed back into the GnRH pulse 
generator. ${ }^{14}$ The arcuate nucleus of the developing brain has been shown to be particularly sensitive to perinatal nutritional conditions. ${ }^{15}$ The sensitive arrangements of neurons found in this region not only are responsible for the complex processes that together initiate the GnRH pulse but also they are intimately involved with the regulation of appetite and energy balance throughout the lifespan.

Early onset of puberty and early nutritional insults are most clearly linked in the research by the activity of two chemical actors, in particular, namely, the metabolic hormone leptin and the peptide kisspeptin. The actions of these chemicals and their receptors are modified by early life nutrition, and both are vital components to the regulation of pubarche. A variety of genetic controls have been theorized to underlie the pattern and timing of this process as a series of hierarchically arranged controls, which ultimately depend on various transcriptional regulatory arrangements, similar to those observed in other gene networks. ${ }^{10}$ These arrangements have been shown to be permanently affected by early environmental cues, programming for a particular phenotypical expression. This enduring programming pattern is termed the 'epigenome', a concept that will be explored more fully later. This epigenetic configuration can alter the action of both leptin and the newly discovered neuropeptide kisspeptin.

The relatively recent discovery of the kisspeptin/ GPR54 system has been hailed the most exciting discovery in this area in the past decade. ${ }^{16}$ The action of kisspeptin has been the focus of a number of studies regarding its role in the onset of puberty. Investigation of the expression and action of this neuropeptide and its receptor units (GPR54) has made it clear that its function is indeed fundamentally involved with the proper timing of puberty onset. Two independent groups of researchers, using genetic knockout models, discovered that kisspeptin acts as a key regulator of the neuroendocrine systems that control the process and that the advent of puberty is the only phenotypical anomaly found in mice and humans lacking functional GPR54 genes coding for kisspeptin receptors. ${ }^{17,18}$ Kisspeptin receives and transmits sex steroid feedback directly into the GnRHproducing cells, acting as a catalyst, and within this system it is indeed a central player in the process underpinning puberty onset. ${ }^{19}$

A recent study by Iwasa et al found that the kisspeptin system is vulnerable to early life malnutrition and demonstrated that intrauterine undernutrition disrupts the production of kisspeptin in rat dams, directly affecting the timing of puberty onset. Furthermore, pubertal timing in the rats becomes normalized with the chronic central injection of kisspeptin. ${ }^{14}$ This compelling model strongly suggests that kisspeptin is a good candidate for the primary regulator of pubarche and that its expression can be disturbed by poor early life nutritional conditions.

Kisspeptin plays an important role in the timing of puberty onset, but it does not act alone. The common adipocyte-derived hormone leptin not only provides the brain with information regarding energy stores in the body but also it is intimately related to the process of puberty onset in various, if not fully understood, ways..$^{20} \mathrm{~A}$ product of the $o b$ gene, leptin is important for various reasons. Mice that do not produce leptin (due to deletion of the $o b$ gene) simply do not undergo puberty, similar to kisspeptin. Puberty is restored with the central chronic introduction of leptin. ${ }^{20}$ Leptin has also been shown to accelerate puberty onset in mice. ${ }^{21}$ It has also been shown to interact with the arcuate nucleus to modify the production of neuropeptide $\mathrm{Y}$, a molecule also involved with energy balance, which has been shown to directly put a brake on the GnRH production in prepubertal stages and has significant effects on the primate neuroendocrine reproductive axis. ${ }^{22,23}$

Serum leptin levels are correlated with birth weight and rise markedly $(50 \%-100 \%)$ just prior to the onset of puberty. As such, leptin was thought of as a somatic signal of adequate energy storage in the form of a critical percentage of fat needed for reproduction. ${ }^{20}$ Leptin levels are no longer generally considered to signal a direct threshold value providing the initiating signal of puberty, but leptin is certainly involved in a very real way. According to at least one group of researchers, levels of soluble leptin receptor may be responsible for the signals that are critical in the regulation and timing of the various developmental stages, including puberty. ${ }^{24}$ Leptin itself is also still considered a necessary player in fertility and an indispensable mediating factor in both the onset of puberty and the energy balance in the body. The body's responsiveness to leptin is certainly impacted by early life nutritional cues, and levels of leptin are known to affect the reproductive ability of women throughout life via various nutritional stresses. ${ }^{7,20,24,25}$

Clearly, the system that underpins the timing of puberty onset is a complex and inter-related process, with the functions of at least two central regulatory chemicals dependent on good maternal and early life nutritional conditions. Further complicating matters, recent findings have shown that leptin can also work as a positive regulator of the kisspeptin/ GPR54 system. Experiments have shown that the kisspeptin system responds poorly in undernourished dams despite increased leptin levels. This may be due to increased leptin 
resistance that develops in undernourished and overnourished offspring, resulting in a partial decoupling of the neurochemical elements controlling this complex system. ${ }^{14}$ Initiation of the observed rise in leptin levels prior to puberty onset, along with the specific actions of kisspeptin and the many other aspects of the co-ordinated chemical cascade that give rise to puberty onset, remains a mystery. ${ }^{20}$ Interestingly, it is generally agreed that discrete epigenetic alterations in gene expression patterns co-ordinate these systems in which fetal and neonatal malnutrition causes characteristic disruptions. ${ }^{10}$ These disruptions can lead to altered onset of puberty and serve as the underlying factors allowing the change in reproductive strategy.

\section{Problems with early puberty}

Precocious puberty occurs when the central nervous system initiates sexual development at an unusually early age, sometimes at an age that may clearly necessitate therapeutic intervention, but, in most cases, it is not cause for any immediate concern. ${ }^{9}$ Early sexual maturity unmatched with social or emotional maturity obviously raises concerns. Less obvious concerns include that, owing to a shortened juvenile growth period, these children will attain an overall shorter stature and have a diminished work capacity. They will also tend to give birth earlier and to smaller babies. ${ }^{7}$

Later in life, more serious problems may await adolescents who present with precocious puberty. In addition to various social and anthropomorphic shortfalls, early puberty has been directly associated with a variety of complex chronic diseases, including adult obesity, development of adult-onset type 2 diabetes, heightened risk of premenopausal breast cancer, cardiovascular disease (CVD), an increase in risktaking behavior, and earlier all-cause mortality. ${ }^{13}$ Parent et $\mathrm{al}^{26}$ suggested that the early onset of puberty could be used as an early sensor for the anomalous interactions of genetics and environmental conditions, echoing Persson et al. ${ }^{2}$ Ahmed et al suggested that the early onset of puberty should be taken very seriously, especially in obese individuals, as a signal of higher risk of CVD. ${ }^{13}$

\section{Studying puberty onset}

It is important to consider the various difficulties in gathering detailed and consistent longitudinal human data regarding the onset of puberty. There are also inherent difficulties with all the varied methods used to determine the exact onset of puberty; some of these methods include monitoring chemical onset (requiring blood sampling), menarche (a definitive but late indicator), breast development (as visual inspection is inaccurate, glandular palpation is required), and monitoring testicular enlargement in males (generally difficult). In an expansive review of variations of puberty onset around the world, Parent et al noted that many studies use various designs and methods, making comparison of findings problematic. ${ }^{26}$ Furthermore, the normal onset of puberty itself varies over time, socioeconomic status, ethnicity, and location, making it a difficult phenomenon to widely generalize. At least one study has linked earlier onset of puberty to the obesity epidemic, but most evidence is equivocal. ${ }^{27}$ Birth date uncertainty is also a significant issue when investigating any age-linked issue in developing countries. The secular trend has been toward lower ages of puberty onset over the last century, seeming to even out in the 1960s among various developed nations. This trend toward earlier puberty continues in developing countries and among different racial and socioeconomic groups in a number of northern nations. As such, the limits to precocious pubarche are subject to periodic local assessment and regular revision. ${ }^{26}$

In investigating this issue, it is important to recall that onset of puberty is a very complex phenomenon that does not occur in a vacuum. Building a model reliant on a single variable, even one as serious as early life nutritional insult, means that the entire picture will not be known. Causality is also an issue. A large Danish study found a relationship between childhood obesity and accelerated onset of puberty, but it did not consider a common cause for both outcomes in a presumably enriched environment. ${ }^{28}$ Timing of puberty onset has a number of other contributing influences, including conventional genetics, life stressors, the geographically determined light-dark rhythm, and exposure to any number of exogenous chemicals. For example, girls who are raised in less supportive families have been shown to undergo accelerated puberty. ${ }^{29}$ Animal trials have shown that exposure to certain common metal pollutants, such as cadmium $\left(\mathrm{Cd}^{2+}\right)$ found in cigarette smoke or manganese in automobile exhaust, also have the capability to accelerate the onset of puberty. ${ }^{30,31}$

Interestingly, accelerated precocious pubarche has been found in many children who have been adopted into Western European homes from different, often poorer environments, suggesting various complex environmental triggers. ${ }^{26}$ Fortunately, with any distributed phenomenon, ranges can still be set for normal and abnormal puberty onset; in fact, it might prove very important as an indicator for the targeted prevention of associated NCD. ${ }^{2,13}$ 


\section{Malnutrition and growth}

Balance of nutritional intake and metabolic output is a central component to the fitness and reproductive success of an organism. An organism better suited to its niche than competitors in this regard has an advantage in passing along its genes, making optimal utilization of available energy supplies a fundamental evolutionary pressure. Malnutrition during these periods may reasonably adjust metabolic and reproductive strategies accordingly. This property, developmental plasticity, exists in virtually all multicellular organisms. ${ }^{32}$ Gluckman and Hanson suggested that the developing human undergoes a plastic phase early in life in which its phenotypical expression is adjusted according to projected environmental conditions; these expression patterns become set as the body size increases and further alterations become too costly. ${ }^{32}$ These individually modified transcription patterns act as a durable link between prenatal nutrition and lifelong gene expression patterns, blending environmental and genomic information into an enduring individual epigenotype.$^{33}$ Insufficient nutrition informs these complicated processes and has various deleterious effects on growth and all aspects of health.

Malnutrition is a broad term that encompasses many forms of nutritional insult. Undernutrition can take place at any stage of life, but it is particularly harmful during fetal development and early childhood. Wasting and stunting are common manifestations of acute and chronic periods of macronutrient malnutrition, respectively. Diagnosis is classically separated into severe acute malnutrition and moderate malnutrition; together, these conditions are implicated in approximately half of all child deaths, owing primarily to the biological synergism of malnutrition and increased vulnerability to infection and other environmental threats. ${ }^{34,35}$ Undernutrition results from the failure to consume proper, adequate supplies of macro- and micronutrients for everyday energy expenditure, regular bodily maintenance, and growth and development, as well as enough to maintain sufficient bodily energy reserves. Technically, malnutrition also encompasses overnutrition, which results in obesity, overstressing bodily capacity to cope with excessive amounts of energy and bringing well-known negative effects over time. In short, proper nutrition is intake balanced with expenditure and is fundamental to overall continued health.

In discussing the impact of early life malnutrition during sensitive programming periods, this article assumes that adequate catch-up growth is attained during the childhood and juvenile stages. This is because, conversely, individuals suffering from chronic, prolonged periods of moderate malnutrition and/or episodes of severe malnutrition in childhood resulting in stunting have shown a delayed rather than accelerated overall development, including delayed onset of puberty, as adequate resources simply are not available to sustain regular childhood growth curves for long periods. ${ }^{36-39}$ Such widespread chronic deprivation is unacceptable in the modern world, ensuring that adequate maternal nutrition and/or proper catch-up growth should be a priority, including routine monitoring and correction of poor childhood growth. Food security is of paramount local and global concern for countless reasons. Easy, cheap, and effective nutritional status indicators (such as mid upper-arm circumference) exist and should be used to monitor childhood growth adequacy in early childhood. These indicators should also be paired with available effective nutritional interventions when growth falters. ${ }^{40}$

Very poor maternal nutrition may result in intrauterine growth restriction (IUGR), in which the fetus is exposed to stresses during development, precluding the achievement of full growth potential. IUGR carries a very serious and direct risk of neonatal morbidity and mortality to the newborn. ${ }^{41} \mathrm{As}$ malnutrition has been a significant selective pressure in our history, we should expect to find complex mechanisms to adapt to such pressures. It is these mechanisms with longterm effects on puberty onset via cues exchanged during critical development windows, not chronic persistent states of malnutrition, that are the subject of this review.

Admittedly, reviewing evidence of delayed development under conditions of chronic deprivation throughout childhood, with evidence of accelerated puberty onset rooted in early life cues, does bring up the question of metabolic adjustments aimed at the normalization of puberty timing under consistent adverse nutritional conditions. There is very little evidence to support this normalization supposition. Cooper et al have determined, at least in a sampling of Western girls, by taking their weights at birth, at 7 years, and at menarche, that the effects of intervening nutrition are statistically independent factors and that in utero programming patterns are maintained, although their study could not consider the effect of long-term chronic nutritional insults on age at pubarche. ${ }^{42}$

\section{The Barker hypothesis and epigenetic programming}

When Lewis et $\mathrm{a}^{43}$ first observed in 1986 that the overfeeding of baboon infants resulted in obesity, which manifested only in adulthood, it gave rise to the question of where the 'memory' of early life nutritional intake is stored for the 
intervening period. ${ }^{44}$ The burgeoning field of epigenetics provides tools to grasp how fetal metabolic memory plays a role in developmental trajectory and disease progression in later life. The first landmark demonstration of the impact of early life nutritional conditions on risk of developing complex adult-onset disorders in humans was by Barker et al. ${ }^{45}$ Focusing on increased ischemic heart disease among low-birth-weight individuals, Barker et al established that environmental factors in early life can cause profound and complex effects on the expression of adult phenotype..$^{45}$ The resulting 'Barker hypothesis' posits that the development of complex NCDs, including diabetes, can be prefigured during certain critical periods early in fetal and neonatal development. Subsequently, this profound effect of early life nutrition on adult phenotype has been reproduced on every continent (excluding Africa). Subsequent data have reinforced that similar early environmental stresses can alter the programming of intracellular signaling and interactions and augment various metabolic pathways throughout the life course. ${ }^{33}$ It must be fully emphasized that these differences stem not from genetic inheritance but rather from early environmental conditions and as such are preventable. ${ }^{46}$

Informed by the Barker hypothesis, the works of Godfrey et al and various other researchers posit that early adjustment of metabolic pathways is informed by various maternal cues. ${ }^{6}$ These maternal cues include diet and body composition before and during pregnancy, levels of stress, as well as fetal, infant, and early childhood nutrition. Certain sensitive processes set up a developmental trajectory, which not only results in earlier puberty onset but also raises the risks of developing various metabolism-related NCDs later in life. ${ }^{6}$ These metabolic effects have been shown to include traits such as a preference for high-fat foods, a pronounced sedentary lifestyle, hypertension, and heightened adiposity. ${ }^{47}$ Frustratingly, in a child trying to attain a normal (catch-up) weight too rapidly, negative effects have been shown to amplify with availability of a hypercaloric nutritional environment in postnatal life. ${ }^{48-50}$ As such, early life interventions must be sought that ameliorate the initial negative influences on the individual phenotype to protect the future health of the subject, and interventions should be implemented carefully with overall health in mind.

It should also be noted that it is not exclusively maternal undernutrition and early life deficits that have been shown to have negative consequences in the longer term; rather, the link between prenatal nutrition, later metabolic disruption, and NCD risk (including diabetes, obesity, and breast cancer) shows a U-shaped distribution spanning the birth weight curve. ${ }^{51,52}$ Under this framework, NCDs are characterized as the end result of decreased responsiveness to new challenges faced by the aging body as a trade-off for earlier sexual maturation. ${ }^{6,32}$

Fortunately, as mentioned earlier, accelerated onset of puberty may have the potential to be used as an early warning sign of heightened risks of developing a number of complex metabolic disorders that seem to stem from related neonatal programming. Understanding this phenomenon may allow for targeted prevention efforts to lessen NCD risks that are ultimately manifested in the lack of adequate responsiveness to external stressors. ${ }^{2,20,53}$

\section{Epigenetic metabolic-reproductive connection}

It has been shown experimentally that gene transcription patterns can be altered by early life nutrition, causing different functioning of at least two of the key fundamental regulating elements underpinning puberty onset, namely kisspeptin and leptin. $10,14,19,20,54$ These chemicals act in the same region of the brain, the arcuate nucleus of the hypothalamus. Maternal nutrition and disrupted macronutrient intake early in life have been linked to permanent changes in gene expression patterns in many animal studies, which have thoroughly proven that maternal dietary, endocrine, or other stresses can indeed induce long-term changes in the cardiovascular and metabolic functioning of offspring. ${ }^{51}$ These early life influences have also been linked to the development of cancer later in life. ${ }^{52,55}$ Using agouti mice, Waterland and Jirtle and Waterland et al demonstrated that even modest changes in maternal nutrient intake can dramatically and permanently affect expressed phenotype by altering genetic expression at specific susceptible epigenetic loci while preserving the genome..$^{56,57}$

Burdge et al have demonstrated experimentally that dietary intake during early development markedly affects gene promoter methylation percentages in rats..$^{53}$ They have suggested that it would require the modification of only a few key transcription promoters, at susceptible loci, to permanently alter the phenotypical expression of a large number of metabolic and developmental pathways. These new epigenetic explanations of the programming observed in sensitive and closely linked neurochemical pathways provide a plausible mechanism for common origins of the early onset of puberty, maladapted energy systems, and subsequent increased disease risk in adulthood. ${ }^{53}$

As any student of biology knows, modification of the underlying genome is primarily driven by random mutation 
and selection pressures, which is a very slow process. Epigenetic modifications are those changes that provide an organism with a method and opportunity for an intermediate, generational change in phenotype without requiring modification of DNA sequencing, thus providing a level of adaptation between selection pressures and homeostasis. Mechanisms behind these changes involve discrete, targeted changes to the pattern of DNA expression via processes such as differing methylation patterns of $\mathrm{CpG}$ sites on the DNA, resulting in the repression of certain gene products, thus modifying phenotype in interesting, progressive, and heritable ways through information based on nutritional influences. ${ }^{56}$

\section{Epigenetic mechanisms}

The regions of DNA where cytosine nucleotides occur next to guanine nucleotides along the length of the DNA strand are called $\mathrm{CpG}$ sites, distinguished from the base pairing of cytosine with guanine: CG. Methylation of cytosine within $\mathrm{CpG}$ dinucleotides at specific sites is key to the gene expression patterns and absolutely crucial to development and cellular differentiation. ${ }^{53}$ Diet-derived methyl donors such as methionine and various cofactors (primarily vita$\min \mathrm{B}_{6}, \mathrm{~B}_{12}$, and folic acid) are required for the synthesis of $\mathrm{S}$-adenosylmethionine, which is required for the subsequent methylation of the $\mathrm{CpG}$ sites. ${ }^{58}$

Configuration of histones (proteins involved in the packaging of DNA) can be modified by various other chemical influences. For instance, lysine residue methylation or acetylation causes a relaxation or condensation of local chromatin structures, occluding or exposing different regions of the DNA to transcription. CpG methylation and altered histone configurations are just two of the most discussed epigenetic processes that are environmentally configured and that add an additional level of control of genetic expression; both the processes can be affected by maternal nutrient intake in early life..$^{53}$

It follows that balanced conditions during early development are critical to the normal onset and progression of puberty and hence optimal adult stature and optimal adult health in an enriched environment, subsequently reducing the chance of development of various NCDs. When looked at through an evolutionary lens, the exchange of long-term health and reproductive longevity for earlier reproductive competence is a good strategy to adopt when presented with difficult nutritional circumstances. Methylation of $\mathrm{CpG}$ sites and alterations in histone configuration provide the mechanism to explain this phenomenon. At least one study has shown that humans who have undergone nutritional insult in utero are not only more prone to metabolic disorders but, under protected conditions, bear more children themselves, suggesting a link between early programming disease risk and fertility. ${ }^{59}$

The fact that the epigenome is configured by maternal environmental and nutritional conditions has led some to suggest that it is perhaps an even more important issue to explore than purely heritable genetic risk..$^{51} \mathrm{~A}$ large body of work exists, largely overlooked by proponents of the theory of modern synthesis, demonstrating a dynamic interaction between the genome and the environment during the plastic phase of development, which also may produce heritable outcomes. ${ }^{60}$

\section{Critical vulnerable periods}

The much-studied tragedy of the 1944 Dutch 'hunger winter' has provided an elegant natural experiment to study the longterm effects of poor maternal nutrition on future disease risk. This event and the longitudinal nature of the studies show conclusively that intrauterine undernutrition increases disease susceptibility later in life. Interestingly, exposure to famine conditions during different stages of gestation had marked different effects on which disease susceptibilities were found, with early gestation showing greatest vulnerability. ${ }^{61}$

Nutritional disturbances occurring early in development tend to damage more elementary systems, such as those associated with nutritional balance, adiposity, blood coagulation, and atherogenic lipid profile, whereas nutritional insults during later gestational periods disrupt higher-order systems, causing problems with kidney function (microalbuminuria) and the respiratory system. ${ }^{61}$ Furthermore, infants exposed to poor environments and poor maternal attention shortly after birth have been found to have disruptions in cognitive and emotional development. ${ }^{62}$ Frienkel's concept of a 'fuel-mediated' teratogenesis throughout the continuum of stages of fetal development is instructive here. Consistent with other research, Frienkel suggests that the developing fetus may be exquisitely attuned to the maternal fuel economy at these key times of cellular proliferation and differentiation. ${ }^{32,58,63}$ Much more data are needed to properly assess the continuity of this phenomenon, the specificity of nutritional insult, and the effects on future health prospects. Nonetheless, there is enough evidence to claim that nutritional imbalances, including excess, at all of these sensitive stages can result in persisting disorders that manifest in later life. All possible efforts should therefore be taken to prevent such insults. 


\section{Public health significance: building maternal capital}

Unprecedented political, scientific, and economic changes have been experienced by the past few generations. The nutritional impacts of the green revolution and industrial food production, on one hand, and the staggering social and physiological consequences of colonialism and economic oppression, on the other hand, have led Wells to introduce the useful public health concept of maternal capital. ${ }^{7}$ This important notion simply considers the mother's phenotypical resources available for investment in offspring. A smaller fetus is clearly less costly to the mother during pregnancy as well as in early life. Such small stature, indicative of poor maternal capital, leads to earlier maturation and affects the development of the next generation. ${ }^{64}$ This theory states that better conditions in early life made available by adequate maternal and early life nutrition allow for improvement of the physiological buffer that is used to protect the offspring from future nutritional insult, thus improving maternal capital and subsequent health of the offspring, and their offspring in turn.

A particularly interesting and potentially controversial ramification of Wells's position is that rapidly rectifying nutritional status of various large groups of people (ie, within a single generation) who have undergone a systematic loss of maternal capital over many generations may unintentionally overload the existing metabolic capacity of offspring. He calls for responsible and moderated policies aimed at not only increasing anthropometric measurements in the short term but also building maternal capital over time, presumably to avoid the Faustian exchange of horribly detrimental maternal and childhood malnutrition for maladapted adults with higher rates of debilitating NCDs. ${ }^{7}$

The modern world continues to undergo various rapid demographic and socioeconomic changes. Many developing countries (India and China, in particular) continue to undergo a rapid nutritional transition, characterized by rapid urbanization, migration, and drastic changes in metabolic balance within a single generation. ${ }^{65}$ Nutritional plentitude unanticipated by early life programming may have particularly dire consequences for families and children who migrate to urban areas and undergo the associated nutritional transition accompanied by reduced physical activity, rapidly stressing a fast programmed metabolism. ${ }^{6}$ Indeed, it can be observed that rapid changes in nutritional balance overload adaptive abilities. Studies have shown that patterns of adiposity are radically changed among previously malnourished people and lead to more centralized patterns of adiposity, diabetes, and CVD in groups who have presumably undergone nutritional insult in early life. We cannot afford to ignore these trends any longer. ${ }^{67}$ India holds the dubious distinction of being 'the diabetes capital of the world', according to the International Diabetes Federation. Asian Indians exhibit greater risk of metabolic disorders, including hypertension, obesity, diabetes, and heart disease, than other ethnic groups. This is tragically almost certainly due to the rapid lifestyle transition brought by the introduction of the Western lifestyle over the last 50 years. Not only are Indians in this explosion of NCD but also Native American groups, Pacific Islanders, and African communities have shown marked increases in metabolic disorders after rapid adoption of Western consumption habits. ${ }^{68}$

Populations in the developing world are experiencing the dual epidemics of communicable disease and NCD as deaths from infectious diseases. Acute maternal and neonatal conditions are in decline, and deaths due to chronic illnesses are projected to increase considerably, accounting for $64 \%$ of all deaths (41 million) by 2015, unless urgent actions are taken. ${ }^{67}$ Unfortunately, concerns about the rising tide of chronic diseases in underdeveloped nations are conspicuously absent from the Millenium Development Goals (MDGs) and hence have not received the attention they deserve on the international stage.$^{69}$ Despite this oversight, greater attention to maternal and child nutrition and improvement of maternal capital could powerfully address the MDGs. Furthermore, improved maternal capital holds the promise to relieve increasing pressures on health care, providing a measure of protection against both acute and chronic diseases in the short and long term.

The world is not going to stop changing anytime soon, and deprived epigenetic programming will continue to translate into poor health in adulthood. The global population is estimated to increase by roughly $40 \%$ over the next 40 years, and increased migration to northern nations (Canada, Scandinavia, Russia, and the northern United States) will be strongly driven by both climatic and economic pressures. ${ }^{70}$ As we have seen, the developing human child is incredibly sensitive to various nutritional cues that hold information about the mother's nutritional conditions, setting a predicted developmental and metabolic trajectory in response to forecasted energy balance for the rest of his/her life. Poor adult health rooted in preventable perinatal conditions in developing regions now will therefore certainly accompany future immigrants to the north in the form of a very large burden of chronic disease risk. Such disease would almost certainly present major challenges to the health care systems 
in the global North. Thus, aside from the very real (yet often sidelined) moral imperative to help vulnerable mothers and children, we should remember that high-quality maternal and early life nutritional interventions and broad initiatives aimed at increasing overall maternal capital among the vulnerable are investments in the future prosperity of the global north, as well as critical to continuing growth and development of economies in the global south. ${ }^{71}$

\section{Conclusion}

It is clear that fetal, neonatal, and early life malnutrition impacts developmental trajectory, puberty onset, and energy balance, and it can also increase the risk of metabolic diseases in adulthood. Therefore, malnutrition during critical developmental windows has the potential to negatively affect virtually all aspects of a child's overall health and development pattern, even after catch-up weight has been achieved, via underlying epigenetic mechanisms. Early nutritional scarcity, or indeed caloric excess in utero, can predispose children to earlier puberty and an array of physical and cognitive disorders.

The importance of ensuring the health and well-being of mothers and young children should not need more evidence to compel rational careful and humane policy creation efforts. Nonetheless, the long-term health ramifications of poorly fed mothers and neonates necessitate more serious investment. The connections are many, and the associated mechanisms have been well explored. The underlying issue of poor maternal capital leading to poor health in adulthood can be mitigated in two ways. First, maternal and early life food security should be carefully protected and paired with early child growth monitoring cheaply and effectively to ensure that vulnerable populations will have a fighting chance to correct the various socioeconomic conditions that perpetuate the cycle of poverty, including depleted maternal capital. Second, further prospective research should be performed to determine whether the onset of puberty can be monitored and local thresholds established for use as an indicator of NCD risk, as both stem from maladaptive early life trauma to the epigenome. If proven, early puberty onset should be met with targeted prevention measures to lessen the growing NCD burdens faced by groups undergoing rapid nutritional transition.

The importance of understanding the various epigenetic mechanisms connecting early life nutrition, puberty onset, and NCD risks cannot be overstated, as such understandings may affect the short-term and long-term health of developing and developed societies. The connection between proper nutrition during early life and good health throughout life should be understood and protected. Realistic plans and resources need to be devoted to prepare to handle the ongoing rise in chronic diseases stemming from rising overnutrition worldwide. These risks may well be heralded by accelerated puberty onset, and more prospective research needs to be done to investigate this phenomenon.

\section{Disclosure}

The author reports no conflicts of interest in this work.

\section{References}

1. Bogin B. Evolutionary perspective on human growth. Annu Rev Anthropol. 1999;28:109-153.

2. Persson I, Ahlsson F, Ewald U, et al. Influence of perinatal factors on the onset of puberty in boys and girls: implications for interpretation of link with risk of long term diseases. Am J Epidemiol. 1999;150(7): 747-755.

3. Gluckman PD, Hanson MA, Buklijas T. A conceptual framework for the developmental origins of health and disease. J Dev Orig Health Dis. 2010;1(1):6-18.

4. Kaati G, Bygren LO, Pembrey M, Sjöström M. Transgenerational response to nutrition, early life circumstances and longevity. Eur $J$ Hum Genet. 2007;15(7):784-790.

5. Gluckman PD, Hanson MA, Beedle AS. Non-genomic transgenerational inheritance of disease risk. Bioessays. 2007;29(2):145-154.

6. Godfrey KM, Gluckman PD, Hanson MA. Developmental origins of metabolic disease: life course and intergenerational perspectives. Trends Endocrinol Metab. 2010;21(4):199-205.

7. Wells JC. Maternal capital and the metabolic ghetto: an evolutionary perspective on the transgenerational basis of health inequalities. $\mathrm{Am}$ J Hum Biol. 2010;22(1):1-17.

8. Pereira ME, Fairbanks LA, editors. Juvenile Primates: Life History, Development, and Behavior. New York: Oxford University Press; 1993.

9. Gluckman PD, Hanson MA. Evolution, development and timing of puberty. Trends Endocrinol Metab. 2006;17(1):7-12.

10. Ojeda SR, Dubay C, Lomniczi A, et al. Gene networks and the neuroendocrine regulation of puberty. Mol Cell Endocrinol. 2010;324(1-2): $3-11$.

11. Marshall WA, Tanner JM. Variations in pattern of pubertal changes in girls. Arch Dis Child. 1969;44(235):291-303.

12. Marshall WA, Tanner JM. Variations in the pattern of pubertal changes in boys. Arch Dis Child. 1970;45(239):13-23.

13. Ahmed ML, Ong KK, Dunger DB. Childhood obesity and the timing of puberty. Trends Endocrinol Metab. 2009;20(5):237-242.

14. Iwasa T, Matsuzaki T, Murakami M, et al. Effects of intrauterine undernutrition on hypothalamic Kiss1 expression and the timing of puberty in female rats. $J$ Physiol. 2010;588(Pt 5):821-829.

15. Wakabayashi Y, Nakada T, Murata K, et al. Neurokinin B and dynorphin A in kisspeptin neurons of the arcuate nucleus participate in generation of periodic oscillation of neural activity driving pulsatile gonadotropin-releasing hormone secretion in the goat. J Neurosci. 2010;30(8):3124-3132.

16. Smith JT, Clarke IJ. Kisspeptin expression in the brain: catalyst for the initiation of puberty. Rev Endocr Metab Disord. 2007;8(1):1-9.

17. Seminara SB, Messager S, Chatzidaki EE, et al. The GPR54 gene as a regulator of puberty. N Engl J Med. 2003;349(17):1614-1627.

18. Funes S, Hedrick JA, Vassileva G, et al. The KiSS-1 receptor GPR54 is essential for the development of the murine reproductive system. Biochem Biophys Res Commun. 2003;312(4):1357-1363.

19. Dunger DB, Ahmed ML, Ong KK. Early and late weight gain and the timing of puberty. Mol Cell Endocrinol. 2006;254-255:140-145. 
20. Mantzoros CS, Flier JS, Rogol AD. A longitudinal assessment of hormonal and physical alterations during normal puberty in boys. V. Rising leptin levels may signal the onset of puberty. J Clin Endocrinol Metab. 1997;82(4):1066-1070.

21. Ahima RS, Dushay J, Flier SN, Prabakaran D, Flier JS. Leptin accelerates the onset of puberty in normal female mice. J Clin Invest. 1997; 99(3):391-395.

22. El Majdoubi M, Sahu A, Ramaswamy S, Plant TM. Neuropeptide Y: a hypothalamic brake restraining the onset of puberty in primates. Proc Natl Acad Sci U S A. 2000;97(11):6179-6184.

23. Finn PD, Cunningham MJ, Pau KY, Spies HG, Clifton DK, Steiner RA. The stimulatory effect of leptin on the neuroendocrine reproductive axis of the monkey. Endocrinology. 1998;139(11):4652-4662.

24. Quinton ND, Smith RF, Clayton PE, et al. Leptin binding activity changes with age: the link between leptin and puberty. JClin Endocrinol Metab. 1999;84(7):2336-2341.

25. Wade GN, Schneider JE, Li HY. Control of fertility by metabolic cues. Am J Physiol. 1996;270(1 Pt 1):E1-E19.

26. Parent AS, Teilmann G, Juul A, Skakkebaek NE, Toppari J, Bourguignon JP. The timing of normal puberty and the age limits of sexual precocity: variations around the world, secular trends, and changes after migration. Endocr Rev. 2003;24(5):668-693.

27. Himes JH. Examining the evidence for recent secular changes in the timing of puberty in US children in light of increases in the prevalence of obesity. Mol Cell Endocrinol. 2006;254-255:13-21.

28. Aksglaede L, Juul A, Olsen LW, Sørensen TI. Age at puberty and the emerging obesity epidemic. PLoS One. 2009;4(12):e8450.

29. Belsky J, Steinberg L, Draper P. Childhood experience, interpersonal development, and reproductive strategy: an evolutionary theory of socialization. Child Dev. 1991;62(4):647-670.

30. Henson MC, Chedrese PJ. Endocrine disruption by cadmium, a common environmental toxicant with paradoxical effects on reproduction. Exp Biol Med (Maywood). 2004;229(5):383-392.

31. Pine M, Lee B, Dearth R, Hiney JK, Dees WL. Manganese acts centrally to stimulate luteinizing hormone secretion: a potential influence on female pubertal development. Toxicol Sci. 2005;85(2): $880-885$.

32. Gluckman PD, Hanson MA. The plastic human. Infant Child Dev. 2010; 19(1):21-26.

33. Dover GJ. The Barker hypothesis: how pediatricans will diagnose and prevent common adult-onset diseases. Trans Am Clin Climatol Assoc. 2009;120:199-207.

34. Pelletier DL, Frongillo EA Jr, Schroeder DG, Habicht JP. The effects of malnutrition on child mortality in developing countries. Bull World Health Organ. 1995;73(4):443-448.

35. Scrimshaw NS, SanGiovanni JP. Synergism of nutrition, infection, and immunity: an overview. Am J Clin Nutr. 1997;66(2):464S-477S.

36. Khan AD, Schroeder DG, Martorell R, Haas JD, Rivera J. Early childhood determinants of age at menarche in rural Guatemala. Am J Hum Biol. 1996;8(6):717-723.

37. Simondon KB, Simondon F, Simon I, et al. Preschool stunting, age at menarche and adolescent height: a longitudinal study in rural Senegal. Eur J Clin Nutr. 1998;52(6):412-418.

38. Kulin HE, Bwibo N, Mutie D, Santner SJ. The effect of chronic childhood malnutrition on pubertal growth and development. Am J Clin Nutr. 1982;36(3):527-536.

39. Satyanarayana K, Naidu AN. Nutrition and menarche in rural Hyderabad. Ann Hum Biol. 1979;6(2):163-165.

40. Connor NE, Manary MJ, Maleta K. Monitoring the adequacy of catch-up growth among moderately malnourished children receiving home-based therapy using mid-upper arm circumference in Southern Malawi. Matern Child Health J. 2010 Jan 16. [Epub ahead of print].

41. Resnik R. Intrauterine growth restriction. Obstet Gynecol. 2002;99(3): 490-496.

42. Cooper C, Kuh D, Egger P, Wadsworth M, Barker D. Childhood growth and age at menarche. Br J Obstet Gynaecol. 1996;103(8):814-817.
43. Lewis DS, Bertrand HA, McMahan CA, McGill HC Jr, Carey KD, Masoro EJ. Preweaning food intake influences the adiposity of young adult baboons. J Clin Invest. 1986;78(4):899-905.

44. Lucas A. Programming by early nutrition: an experimental approach. J Nutr. 1998;128(2 Suppl):401S-406S.

45. Barker DJ, Osmond C, Law CM. The intrauterine and early postnatal origins of cardiovascular disease and chronic bronchitis. $J$ Epidemiol Community Health. 1989;43(3):237-240.

46. Kramer MS. Determinants of low birth weight: methodological assessment and meta-analysis. Bull World Health Organ. 1987;65(5):663-737.

47. Bellinger L, Lilley C, Langley-Evans SC. Prenatal exposure to a maternal low-protein diet programmes a preference for high-fat foods in the young adult rat. Br J Nutr. 2004;92(3):513-520.

48. Adair LS. Size at birth predicts age at menarche. Pediatrics. 2001; 107(4):E59.

49. Vickers MH, Breier BH, McCarthy D, Gluckman PD. Sedentary behavior during postnatal life is determined by the prenatal environment and exacerbated by postnatal hypercaloric nutrition. Am J Physiol Regul Integr Comp Physiol. 2003;285(1):R271-R273.

50. Vickers MH, Breier BH, Cutfield WS, Hofman PL, Gluckman PD. Fetal origins of hyperphagia, obesity, and hypertension and postnatal amplification by hypercaloric nutrition. Am J Physiol Endocrinol Metab. 2000;279(1):E83-E87.

51. Gluckman PD, Hanson MA, Cooper C, Thornburg KL. Effect of in utero and early-life conditions on adult health and disease. $N$ Engl J Med. 2008;359(1):61-73.

52. dos Santos Silva I, de Stavola BL, Hardy RJ, Kuh DJ, McCormack VA, Wadsworth ME. Is the association of birth weight with premenopausal breast cancer risk mediated through childhood growth? $\mathrm{Br} J$ Cancer. 2004;91(3):519-524.

53. Burdge GC, Hanson MA, Slater-Jefferies JL, Lillycrop KA. Epigenetic regulation of transcription: a mechanism for inducing variations in phenotype (fetal programming) by differences in nutrition during early life? Br J Nutr. 2007;97(6):1036-1046.

54. Sugden MC, Langdown ML, Munns MJ, Holness MJ. Maternal glucocorticoid treatment modulates placental leptin and leptin receptor expression and materno-fetal leptin physiology during late pregnancy, and elicits hypertension associated with hyperleptinaemia in the early-growth-retarded adult offspring. Eur J Endocrinol. 2001;145(4): 529-539.

55. Harder T, Bergmann R, Kallischnigg G, Plagemann A. Duration of breastfeeding and risk of overweight: a meta-analysis. Am J Epidemiol. 2005;162(5):397-403.

56. Waterland RA, Jirtle RL. Transposable elements: targets for early nutritional effects on epigenetic gene regulation. Mol Cell Biol. 2003; 23(15):5293-5300.

57. Waterland RA, Lin JR, Smith CA, Jirtle RL. Post-weaning diet affects genomic imprinting at the insulin-like growth factor 2 (Igf2) locus. Hum Mol Genet. 2006;15(5):705-716.

58. Van den Veyver IB. Genetic effects of methylation diets. Аnnu Rev Nutr. 2002;22:255-282.

59. Painter RC, Westendorp RG, de Rooij SR, Osmond C, Barker DJ, Roseboom TJ. Increased reproductive success of women after prenatal undernutrition. Hum Reprod. 2008;23(11):2591-2595.

60. Gluckman PD, Hanson MA, Beedle AS. Principles of Evolutionary Medicine. New York: Oxford University Press; 2009.

61. Roseboom T, de Rooij S, Painter R. The Dutch famine and its long-term consequences for adult health. Early Hum Dev. 2006;82(8):485-491.

62. Fish EW, Shahrokh D, Bagot R, et al. Epigenetic programming of stress responses through variations in maternal care. Ann NY Acad Sci. 2004; 1036:167-180.

63. Freinkel N. Banting lecture 1980. Of pregnancy and progeny. Diabetes. 1980;29:1023-1035.

64. Hokken-Koèlega AC. Timing of puberty and fetal growth. Best Pract Res Clin Endocrinol Metab. 2002;16(1):65-71. 
65. Misra A, Singhal N, Khurana L. Obesity, the metabolic syndrome, and type 2 diabetes in developing countries: role of dietary fats and oils. J Am Coll Nutr. 2010;29(3 Suppl):289S-301S.

66. Misra A, Khurana L. Obesity and the metabolic syndrome in developing countries. J Clin Endocrinol Metab. 2008;93(11 Suppl 1):S9-S30.

67. World Health Organization. Preventing Chronic Diseases: A Vital Investment. Geneva: World Health Organization; 2005.

68. Robinson R. The fetal origins of adult disease: no longer just a hypothesis and may be critically important in South Asia. BMJ. 2001;322(7283):375-376.
69. Fuster V, Voûte J. MDGs: chronic diseases are not on the agenda. Lancet. 2005;366(9496):1512-1514.

70. Smith LC. The World in 2050: Four Forces Shaping Civilization's Northern Future. New York: Dutton; 2010.

71. Mohan V, Sandeep S, Deepa R, Shah B, Varghese C. Epidemiology of type 2 diabetes: Indian scenario. Indian J Med Res. 2007;125(3): 217-230.

\section{Publish your work in this journal}

Adolescent Health, Medicine and Therapeutics is an international, peer-reviewed, open access journal focusing on health, pathology, and treatment issues specific to the adolescent age group. All aspects of health maintenance, preventative measures and disease treatmen interventions are addressed within the journal and practitioners from all disciplines are invited to submit their work as well as healthcare researchers and patient support groups.. The manuscript management system is completely online and includes a very quick and fair peerreview system. Visit http://www.dovepress.com/testimonials.php to read real quotes from published authors.

Submit your manuscript here: http://www.dovepress.com/adolescent-health-medicine-and-therapeutics-journal 